\title{
Préparation de mélange homogène et stratifié en injection directe dans un moteur à allumage commandé
}

\author{
A homogeneous and laminated mixture for direct injection \\ in a controlled ignition engine
}

par M. Pontoppidan

Magneti Marelli France

The first part of the paper outlines the main reasons for which a G DI system offers a potential solution to meet the future EURO 2000 legislation and describes the main system structure of such a concept. In the second part of the paper is presented a combined experimental and numerical simulation approach concept for optimisation of the charge formation in the combustion chamber. The approach is based on the simultaneous use of engine test rig experiments, detailed visualisation and analysis of injector spray formation and intensive use of $3 D$ multiphase numerical simulation.

The paper concludes with some suggestions for useful work to be done in a nearby future in order to improve the computational and experimental optimisation tools.

\section{I $\square$ INTRODUCTION}

Les exigences de la part des pouvoirs publics et du client/utilisateur, en matière de prix, de prestations et de propreté du véhicule propulsé par un moteur à allumage commandé, sont en évolution croissante. Cela oblige l'ingénieur chargé du développement du moteur, au fur et à mesure que le contexte industriel le lui impose, de conduire des recherches de base de plus en plus complexes, notamment dans le domaine de la préparation de la charge air/combustible destinée à être brûlée dans la chambre de combustion du moteur à allumage commandé.

La nouvelle législation EURO 2000 prévoit déjà une réduction supplémentaire en matière des polluants classiques du moteur à allumage commandé, telle que l'oxyde de carbone $(\mathrm{CO})$, les hydrocarbures imbrûlés $(\mathrm{HC})$ et les oxydes d'azote $\left(\mathrm{NO}_{5}\right)$, mais introduirait probablement également des limitations sévères en ce qui concerne la quantité de dioxyde de carbone émise, dans le but de décroître la consommation spécifique des moteurs thermiques et par ce biais réduire l'impact de «l'effet de serre ».

Une certaine réduction de la consommation spécifique peut déjà être obtenue en faisant travailler le moteur à allumage commandé en conditions, dites de mélange pauvre (richesse $<0,7$ ), en utilisant des systèmes classiques d'injection indirecte dans le collecteur d'air en amont des soupapes d'admission. Si ce système de préparation de mélange est de type séquentiel et utilise des injecteurs à assistance pneumatique à la pulvérisation ainsi que des taux important de renvoi à l'admission des gaz brûlés (EGR) et éventuellement un contrôle variable de la distribution (VVT), un gain de l'ordre de $10 \%$ en consommation spécifique est possible. Or, le fonctionnement en charge partielle du moteur équipé d'un système d'injection indirecte est limité au concept de mélange pauvre à préparation homogène à cause des critères d'agrément de conduite aujourd'hui jugés acceptables par l'utilisateur du véhicule. Si des réductions substantielles supplémentaires sont exigées, le moteur à allumage commandé est forcé de travailler en conditions, dites de pauvreté extrême (richesse $<0,3$ ), ce qui donne la possibilité de faire fonctionner le moteur sans un organe de dosage de l'air (corps de papillon) et ainsi supprimer les pertes par pompage. Cependant, pour rendre possible ce type de fonctionnement, le seul concept de préparation de mélange compatible est celui d'une stratification extrême de la charge dans la chambre de combustion, ce qui peut être obtenu par une injection tardive dans la phase de compression du cycle de Beau de Rochas (environ 60 à $40^{\circ}$ vilebrequin avant le point mort haut $(\mathrm{PMH})$ ). Un dispositif qui peut, de manière efficace et avec des variations cycliques faibles, contrôler une préparation de mélange hautement stratifiée, est celui basé sur le concept d'injection directe à essence (GDI) dans la chambre de combustion.

\section{CONCEPTS DE PRÉPARATION DE MÉLANGE EN GDI}

Déjà dans la phase d'approche du développement d'un système d'injection directe à essence, et surtout quand il s'agit 
d'un fonctionnement en mélange pauvre ou extrêmement pauvre, il devient rapidement évident que la qualité de la combustion est très influencée par la géométrie de la chambre, la phase et la durée de l'injection et la géométrie et le comportement dynamique du jet produit par l'injecteur. La vitesse de pénétration dans le gaz environnant du jet et sa granulométrie sont deux paramètres fondamentaux du processus de mélange qui conditionne la formation de la charge finale présente dans la chambre au moment du départ de la boule de feu dans l'entrefer de la bougie.

De manière générale, une vitesse élevée de pénétration du jet provoque un éclatement violent (« splashing ») des gouttelettes sur la surface supérieure du piston et sur les parois de la chambre et dans des cas extrêmes induit des problèmes sérieux d'accumulation de combustible dans l'espace annulaire entre le piston et la paroi de la chambre au-dessus des segments. Cependant, ce phénomène peut être largement modifié par la volatilité du jet même, laquelle est conditionnée par la granulométrie et la quantité de mouvement des gouttelettes. C'est pourquoi un contrôle très précis du processus d'interaction dynamique entre la formation du jet et les mouvements des parties solides ou gazeuses présentes dans la chambre est primordial pour obtenir une optimisation de la phase de préparation du mélange à brûler afin d'aboutir à une qualité de combustion satisfaisante.

Comme le montre la figure $I$, on peut distinguer deux différents types de conditionnement de l'interaction entre le jet produit par l'injecteur et son environnement. Le concept, dit à guidage solide, utilise activement la forme géométrique de la partie supérieure du piston pour faire dévier les parties liquides les plus lourdes du jet dans une direction souhaitée. En effet, si un injecteur, doté d'un pulvérisateur produisant une vitesse de pénétration élevée, est utilisé, il est indispensable de baser le dessin de la chambre sur le concept de guidage solide. Si par contre le pulvérisateur de l'injecteur a été conçu pour produire des gouttelettes ayant une vitesse et une quantité de mouvement basse, le concept de guidage par aérométrie est très bien adapté, car les mouvements de rotation interne au gaz dans le sens axial de la chambre (tumble) ou perpendiculaire à l'axe (swirl) effectuent un mélange et/ou une stratification très efficace. En fonction du type de fonctionnement du moteur, il peut être opportun d'effectuer le dessin de la chambre de manière à réunir les aspects des deux concepts afin de trouver un compromis qui couvre de façon optimale, et la préparation maigre stratifiée en charge réduite et la préparation homogène et stoechiométrique en pleine charge.

\section{III $\square$ CONDITIONS MÉCANIQUES AUX LIMITES}

Pour mieux comprendre les conditions aux limites du circuit hydraulique, qui produit et contrôle par moyens électro- niques le jet de combustible, une brève description du schéma type de réalisation industrielle est donnée ci-dessous.

La figure 2 montre le schéma synoptique du système GDI de Magneti Marelli pour un moteur à 4 cylindres doté d'une rampe commune (3), avec capteur de pression instantanée incorporé, qui alimente les injecteurs (4). La rampe à son tour est alimentée par une pompe mécanique (2) à pression variable contrôlée par une vanne à régulation électronique rapide $\left(3 \mathrm{Mpa}<P_{i}<12 \mathrm{Mpa}\right.$ ). La raison pour laquelle un concept de pression variable est nécessaire est l'impératif d'assurer une dynamique linéaire de débit de l'injecteur de l'ordre de 20, afin de satisfaire à la fois les exigences de débit en mélange pauvre stratifié (charge partielle avec injection tardive près du $\mathrm{PMH}$ ) et en mélange homogène stratifié (pleine charge) avec des rapports cycliques d'ouverture (RCO) de l'injecteur extrêmement courts (de l'ordre de la milliseconde). Etant donné que la pression d'alimentation ne dépasse pas les $15 \mathrm{Mpa}$ le concept de fonctionnement électromagnétique de l'obturateur de l'injecteur est encore acceptable pour un compromis optimisé de prestations, d'encombrement et de prix de fabrication.

Comme évoqué ci-dessus, la qualité de la combustion, et avec celle-ci la performance du moteur, est étroitement liée au processus de pulvérisation dans le cylindre, qui pour une géométrie solide donnée de la chambre de combustion, est conditionné par les caractéristiques spatiales et temporelles du jet produit par le pulvérisateur de l'injecteur. Pour pouvoir couvrir le plus grand nombre de figures de montage (montage central ou latéral dans la chambre) et une gamme étendue de vitesses de pénétration, trois différents types de dessins de pulvérisateur sont actuellement utilisés. La figure 3 donne un aperçu de leurs principes ayant les caractéristiques principales suivantes relevées à une pression d'alimentation (amont) de $12 \mathrm{Mpa}$, une pression ambiante de décharge (aval) de 0,1 Mpa et une température ambiante de $298^{\circ} \mathrm{K}$ :

- L'injecteur à téton mono-jet à cône creux. Angle primaire de cône entre 30 et $90^{\circ}$. Vitesse moyenne de pénétration sur l'axe du jet de $90 \mathrm{~ms}$ (dispersion de 30 à $150 \mathrm{~m} / \mathrm{s}$ ). SMD (Sauter Mean Diameter) en liquide de test d'injecteurs de 18 à $23 \mu \mathrm{m}$ à $30 \mathrm{~mm}$ du nez de l'injecteur.

- L'injecteur à adaptateur de rotation (swirl) ayant entre 2 et 5 (cas plus fréquent 4) canaux de mise en rotation en amont du calibreur. Le jet est également de type cône creux avec un mouvement rotationnel autour de l'axe du jet. Angle primaire de cône entre 45 et $110^{\circ}$. Vitesse moyenne axiale de pénétration de $60 \mathrm{~m} / \mathrm{s}$ (dispersion de 20 à $100 \mathrm{~m} / \mathrm{s}$ ). SMD en liquide de test d'injecteurs de 20 à $30 \mu \mathrm{m}$ à $30 \mathrm{~mm}$ du nez de l'injecteur.

- L'injecteur à capuchon fermé doté de plusieurs trous ou une fente permettant une direction principale des jets ou nappe de combustible à un angle différent de celui de l'axe

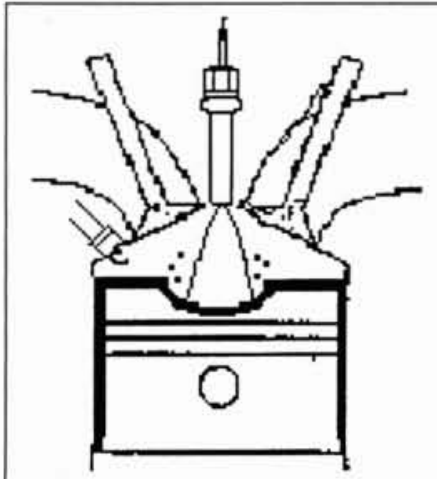

Guidage solide

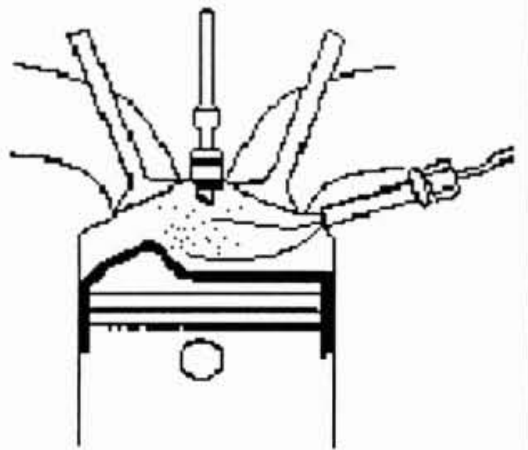

Guidage par aérométrie
1. Concepts de préparation de mélange. 


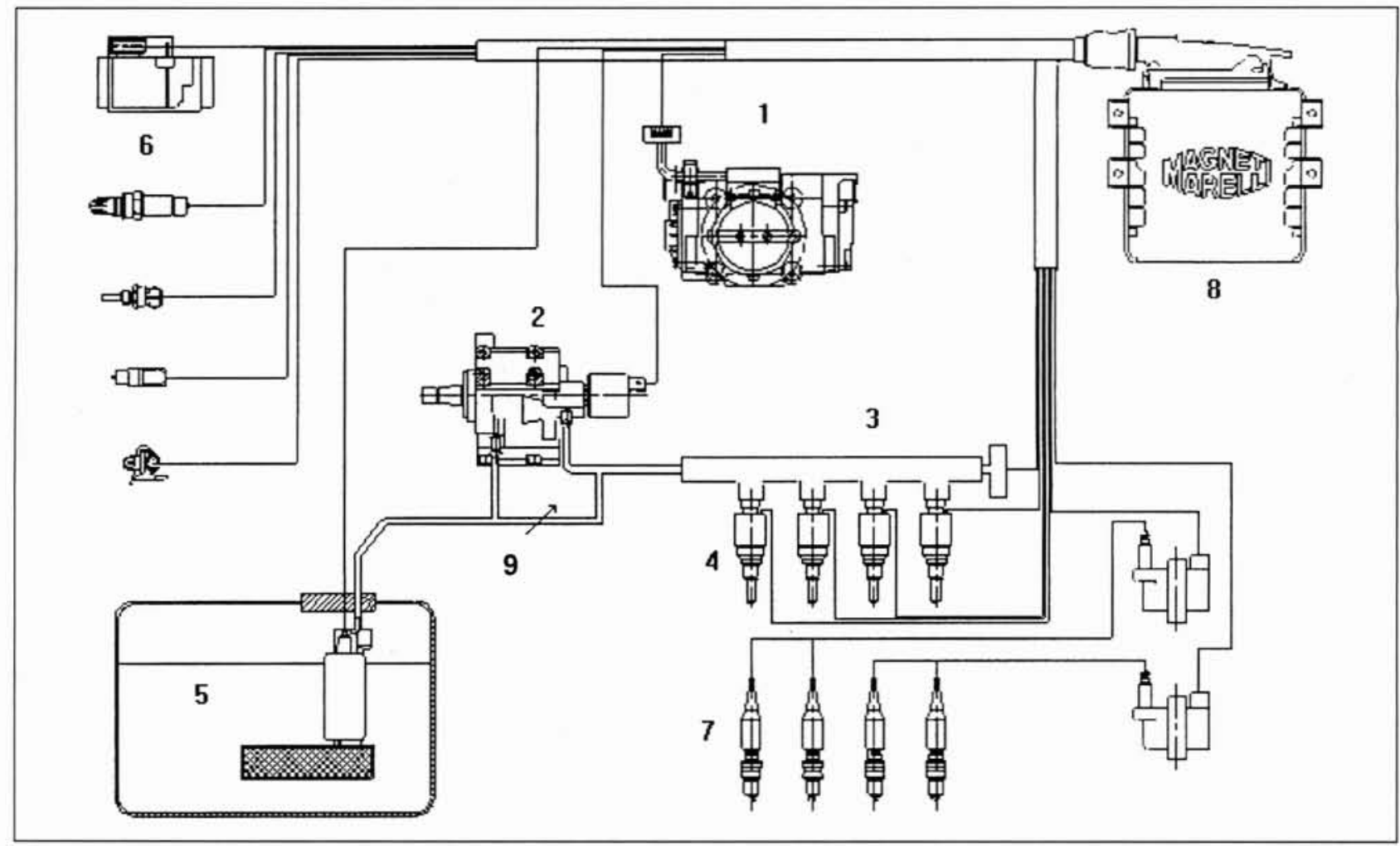

1. Corps de papillon motorisé - 2. Pompe haute pression - 3. Rampe à essence - 4. Injecteurs - 5. Réservoir d'essence 6. Capteurs - 7. Bougies - 8. Calculateur électronique - 9. By-pass.

2. Schéma synoptique de système de GDI.

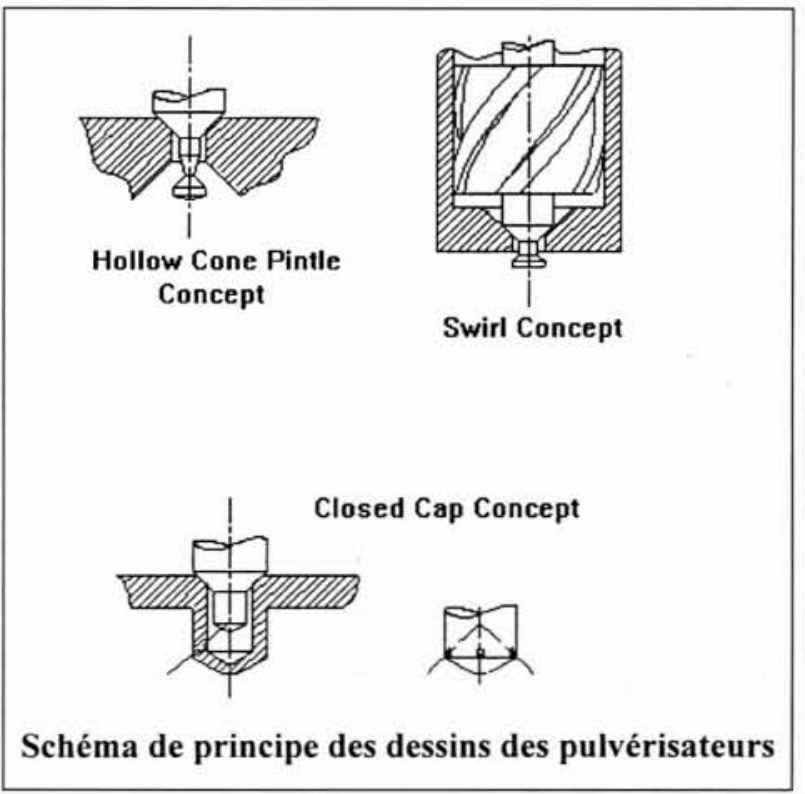

3. Schéma de principe des dessins des pulvérisateurs.

de l'injecteur. En version «trous » plusieurs jets coniques solides sont produits, dans le cas «fente » une nappe mince est produite. Vitesse moyenne dans la direction principale d'éjection à $30 \mathrm{~mm}$ du nez de l'injecteur de $40 \mathrm{~m} / \mathrm{s}$ (dispersion de 15 à $70 \mathrm{~m} / \mathrm{s}$ ). SMD en liquide de test d'injecteurs de 20 à $35 \mu \mathrm{m}$ à $30 \mathrm{~mm}$ du nez de l'injecteur.

\section{IV — MOYENS D'EXPÉRIMENTATION ET DE CALCUL}

Vu l'extrême complexité de l'interactivité spatiale et temporelle entre la formation du jet et l'aérométrie dans la chambre, une utilisation simultanée et des moyens performants d'expérimentation et de visualisations à l'échelle réelle, sans et avec combustion, et des moyens de calcul par simulation numérique en 3D est absolument nécessaire. Une brève énumération des outils utilisés est donnée ci-dessous.

\subsection{Banc de débit à haute pression}

Un banc spécifique de haute pression (fig. 4) a été réalisé dans le but de pouvoir effectuer, en conditions stationnaires et répétitives, des visualisations et des relevés optiques des caractéristiques géométriques et temporelles du jet produit par l'injecteur. La pression d'alimentation en combustible est réglable entre $0,3 \mathrm{MPa}$ et $15 \mathrm{MPa}$. L'injecteur est monté dans une enceinte pressurisable entre $-0,07 \mathrm{MPa}$ et $0,8 \mathrm{MPa}$ par rapport à l'atmosphère et dotée des accès optiques permettant la visualisation et des relevés optiques du jet. Le banc est équipé des moyens nécessaires au contrôle dynamique de l'injecteur en fréquence et en RCO. Les moyens optiques utilisés pour la visualisation du processus de formation du jet utilisent des sources d'éclairage qui sont de forte puissance continue ou par éclat phasé en 3D ou 2D (tomographie laser et enregistrement Schlieren). L'éclairage continu est utilisé principalement pour la cinématographie rapide, tandis qu'en éclat phasé les moyens d'enregistrements sont des caméras CCD (Charge Coupled Device). Les relevés des champs de vitesses dans le jet sont effectués en PIV (Particle Image Velocimetry) ou en technique de Phase 


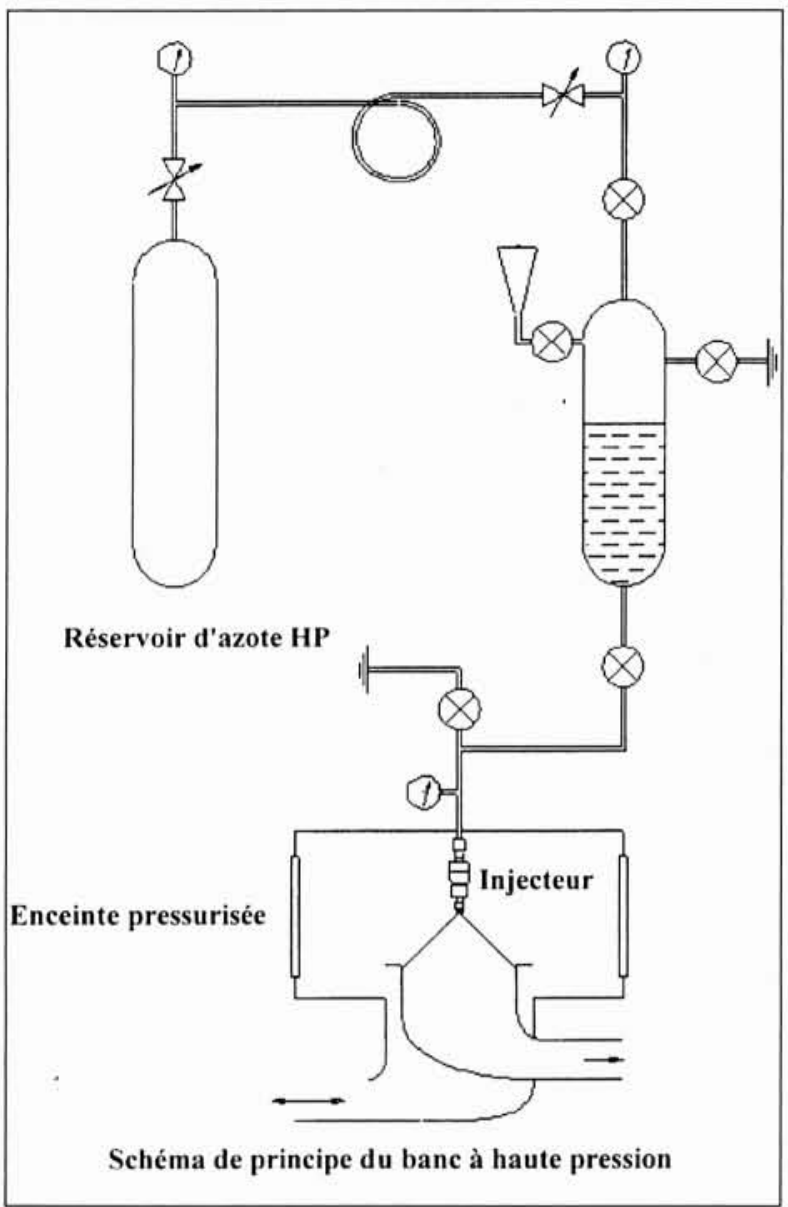

4. Schéma de principe du banc à haute pression.

Doppler, ce dernier offrant également la possibilité de mesures de granulométrie. La figure 5 donne un exemple d'un enregistrement Schlieren effectué dans l'enceinte à $0,8 \mathrm{MPa}$ de contrepression et $570^{\circ} \mathrm{K}$.

\subsection{Moteur mono-cylindre transparent}

Avec le banc moteur multi-cylindre classique, le moteur mono-cylindre doté des accès optiques directs à la chambre de combustion constitue un moyen d'investigation très important pour comprendre les phénomènes physiques et thermodynamiques ayant lieu durant les phases de prépara-

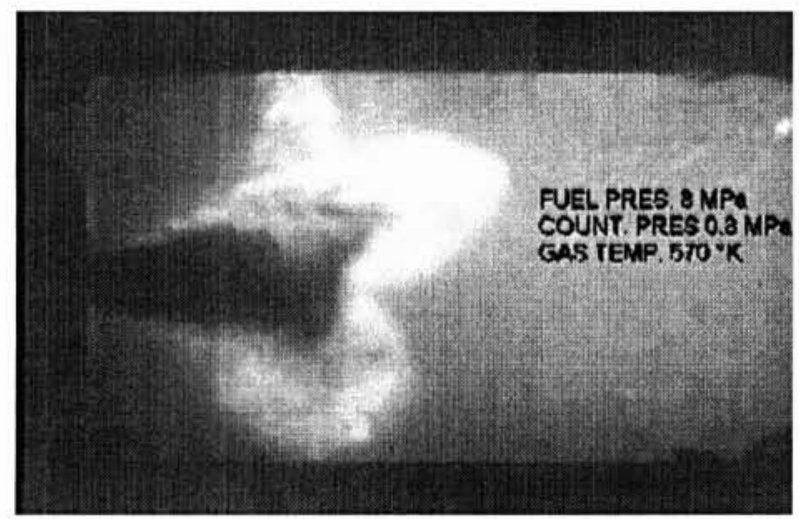

5. Enregistrement Schlieren dans l'enceinte pressurisée.

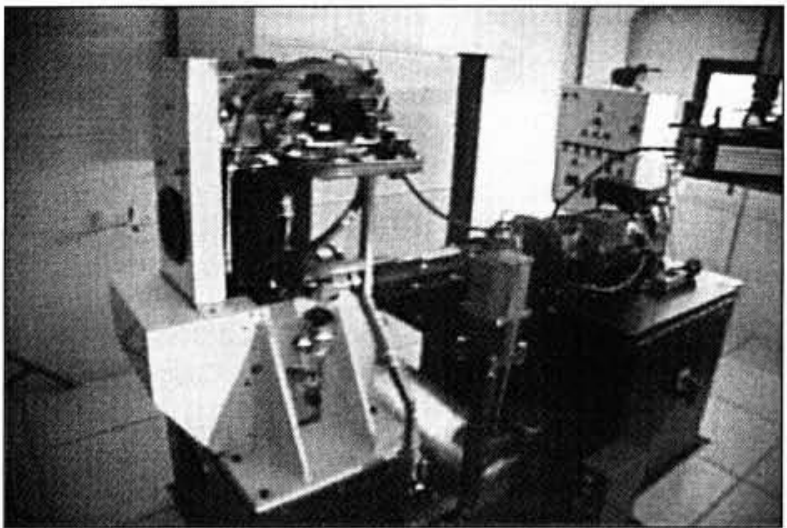

6. Ensemble moteur mono-cylindre transparent.

tion de la charge et sa combustion. Le moteur (fig. 6) peut fonctionner soit en mode entraîné (sans combustion), soit en mode avec allumage et combustion. L'accès optique principal est obtenu en séparant la partie haute et la partie basse du piston. Comme le montre la figure 7 , par le biais de cette séparation il est possible d'intercaler un miroir incliné sur l'axe du piston par lequel la chambre de combustion peut être observée à travers la surface supérieure du piston réalisée en quartz transparent. Ainsi des informations détaillées peuvent être récoltées sur, entre autres, les vitesses de propagation de front de flammes dans la chambre en fonction de la préparation de la charge qui a précédé le début de la combustion. Ces observations sont très importantes pour la validation des codes de calcul. Il est également possible en mode entraîné de substituer la chemise métallique du cylindre par une en quartz transparent et ainsi permettre un accès perpendiculaire à l'axe du cylindre près de la culasse pour la tomographie laser ou l'anémométrie Phase Doppler dans le jet jaillissant de l'injecteur.

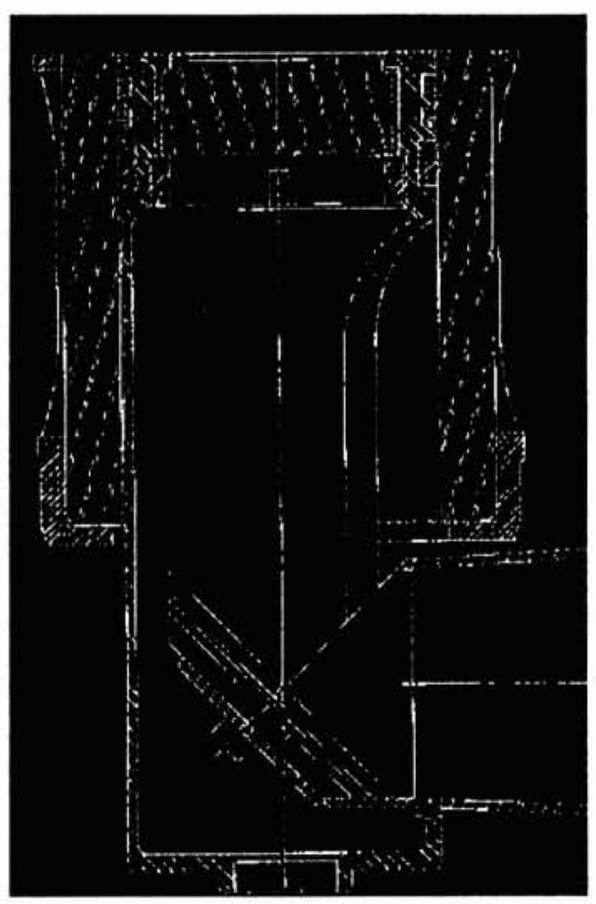

7. Détail de l'accès optique. 


\subsection{Simulation numérique par calcul 3D}

En ce qui concerne l'approche du problème de la formation de la charge par calcul numérique, le cadre général fonctionne jusqu'à un certain point de manière satisfaisante. Un certain nombre de codes commerciaux existent sur le marché (STAR/SPEED/FIRE), mais souffrent un peu du fait d'un manque de transparence surtout au niveau des sous-modèles et de leurs structures pour des motifs commerciaux évidents. C'est pourquoi nous prenons pour la description de l'état d'avance de l'art comme exemple le code NCF-3D développé par Magneti Marelli avec l'université de Rome, car les performances et les lacunes de ce code sont assez similaires à ce que l'on trouve en utilisant les trois codes commerciaux précités sur des cas tests communs. Le code NCF-3D est basé sur le code KIVA-3V, à l'origine développé par Los Alamos Laboratories [1]. Sa structure permet le calcul d'un écoulement 3D, non stationnaire avec turbulence, compressible, en multi-phase et chimiquement réactif dans un milieu ambiant constitué par une géométrie solide en mouvement. L'intégration des équations de Navier-Stoke couplées avec les équations de turbulence, s'effectue par l'utilisation d'une technique à volumes finis utilisant un schéma de calcul arbitraire de Lagrange et d'Euler (ALE = Arbitrary Lagrangean Eulerian). L'intégration numérique est effectuée dans une structure multi-bloc à grille d'hexaèdre. Les sous-modèles spécifiques, suivants, jugés nécessaires pour la modélisation de la physique du concept GDI, ont été ajoutés au solveur général :

- L'introduction des caractéristiques expérimentales du jet au point de jaillissement initial (angle primaire du jet, axe principal de propagation, diamètres initiaux des gouttelettes et leurs emplacements dans le jet, vitesses initiales des gouttelettes),

- Une loi de transfert de chaleur entre le gaz et les parois,

- Une loi d'interaction gouttelettes/parois,

- Un sous-modèle d'initiation de la boule de feu dans l'entrefer de la bougie,

- Loi spécifique pour l'échelle de temps de la longueur turbulente dans le front de flamme,
- Sous-modèle de combustion (iso-octane) de type «flamelet ",

- Sous-modèle de simulation d'auto-allumage des hydrocarbures de type « shell».

Les détails spécifiques de ces modèles ont été décrits dans les publications suivantes $[2,3,4,5]$. Un exemple d'un calcul effectué avec le code NCF-3D pour un injecteur à téton monté sur le côté de la chambre est montré par la figure 8. A un angle de villebrequin de $20^{\circ}$ après le PMH la situation est montrée en ce qui concerne la nappe stoechiométrique non encore brûlée (à gauche sur la figure) et des surfaces d'isotempérature dans la zone de passage du front de flamme (à droite sur la figure).

\section{DÉVELOPPEMENTS UTILES À COURT ET MOYEN TERME}

Compte tenu de l'état actuel des divers projets de moteurs alimentés en injection directe à essence qui, pour la première génération, sont prévus pour une sortie sur le marché en quantité réduite dans l'année 2000 , il serait opportun de focaliser les réflexions de développement ultérieur dans les directions suivantes : l'optimisation technologique encore plus poussée des pulvérisateurs de l'injecteur et le perfectionnement, d'un côté du type de solveur utilisé pour les simulations numériques et, de l'autre côté, de certains sousmodèles déjà implantés sous des formes trop simplistes. De manière plus précise les deux directions peuvent être brièvement décrites comme suit.

En ce qui concerne le dessin des pulvérisateurs, il est certain qu'une recherche vers une plus grande stabilité cyclique de la géométrie du jet/nappe ainsi qu'une population granulométrique plus serrée tout en ayant des gouttelettes à faible quantité de mouvement est une avancée très utile. Cependant, afin de maintenir un injecteur «bon marché » de type à commande électromagnétique, il faut s'assurer que ces dessins n'imposent pas des contraintes de pertes internes hydrauliques élevées, qui dégradent les prestations temporelles de l'injecteur. Compte tenu de la nature du combus-

8. Nappe stoechiométrique et surfaces iso-température à 20 après le PMH.

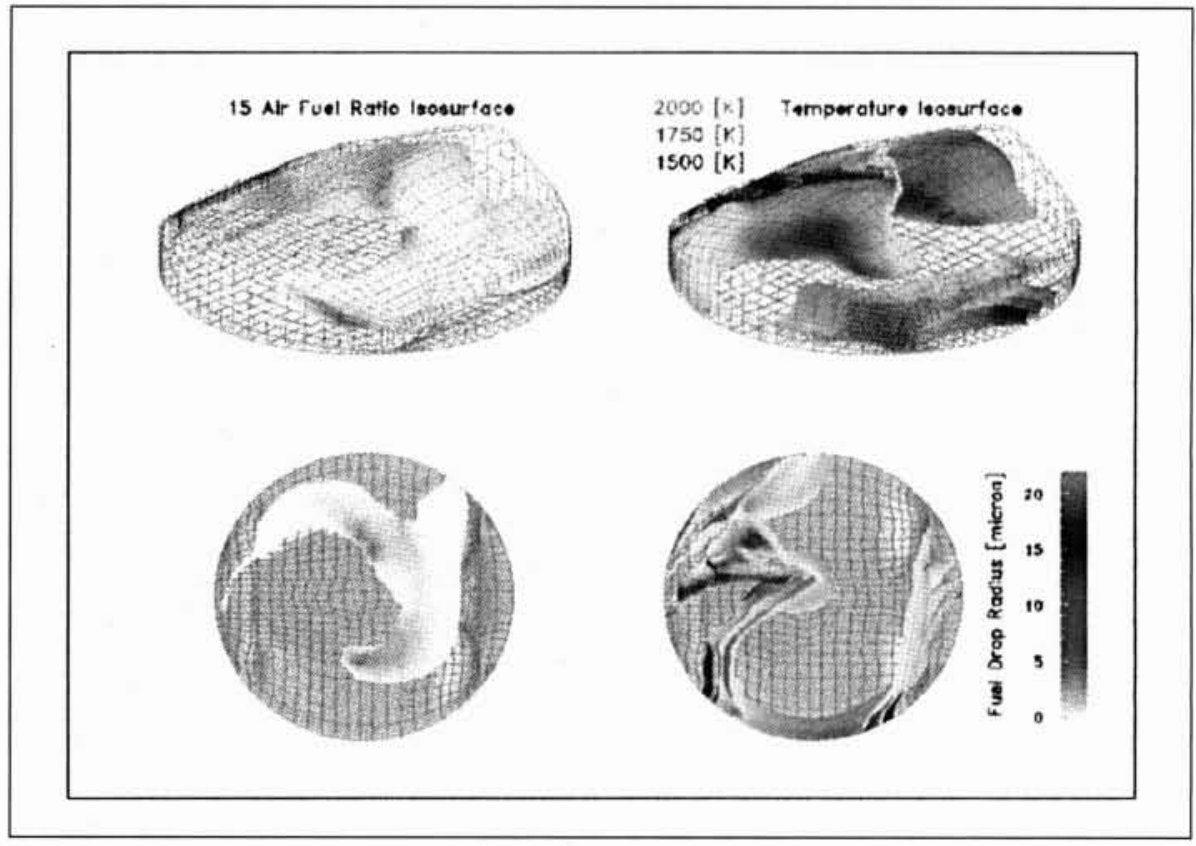


tible et des températures auxquelles l'injecteur est soumis, les phénomènes éventuels de cavitation interne au niveau du calibrage de l'injecteur sont à l'heure actuelle peu connus.

Dans le domaine du calcul numérique, il est certain que le développement d'un solveur adapté en grille non structurée est absolument nécessaire, car la situation actuelle des systèmes multi-blocs à grille structurée ne permet pas, de manière aisée, de changer rapidement la géométrie d'une culasse ou l'emplacement d'un injecteur dans la zone des soupapes, car tout mouvement non axial dans la grille structurée est susceptible de créer, à terme, des déformations de cellules trop importantes. Des sous-modèles fiables de «break-up " d'un jet jaillissant d'un pulvérisateur complexe serait également une chose intéressante par rapport à la situation actuelle où seulement la voie d'introduction des valeurs mesurées est utilisable. Liée à ce phénomène est également la prise en compte au niveau des modelés de vaporisation et de combustion d'un combustible d'une complexité supérieure à celle de l'iso-octane avec une courbe complexe et fractionnée de destination. Cependant, dans ce domaine, un compromis entre les exigences en temps de calcul et la complexité souhaitée doit être effectué afin de garantir une utilisation industrielle du code de calcul.

\section{RÉFÉRENCES}

[1] Amsden A.A. (1993), - « Kiva III : A Kiva program with Block-Structured Mesh for Complex Geometries », Los Alamos Labs, LS 12503 MS.

[2] PONTOPPIDAN M., Bella G. and Rocco V. - « Mixture formation in SI-Engine intake Systems. A simplified theoretical Model with Experimental Verification ». ASME 1996 Spring Congress.

[3] PONTOPpidan M., Gaviani G., Bella G., Rocco V. « Direct Fuel Injection. A Study of Injector Requirements for Different Mixture Preparation Concepts », SAE Paper 970628.

[4] PONTOPpidan M., Gaviani G., Bella G., Rocco V. « Experimental and Numerical Study of Fuel Spray Behaviour to Optimize Charge Motion in Direct Injected SI Engine », ICE 97, International Engines Symposium, Capri.

[5] YANG J., MARTIN J.K. (1989), - « Approximate Solution of One Dimensional Energy Equations for Transient Compressible Low Mach Number Turbulent Flow », Journal of Heat and Mass Transfer, vol. 1, p. 619. 